\title{
Erratum: Correlation control for pure and efficiently generated heralded single photons [Phys. Rev. A 91, 013819 (2015)]
}

\author{
Jefferson Flórez, Omar Calderón, Alejandra Valencia, and Clara I. Osorio \\ (Received 24 November 2016; published 7 December 2016)
}

DOI: 10.1103/PhysRevA.94.069901

In our original paper, we incorrectly labeled the curves in Figs. 11(b) and 12(b). As a consequence, the bandwidth of the filters considered for each curve cannot be read directly from the original figures. Below, we present the figures with the correct labels. The changes in the labels do not affect the overall conclusion of the original paper.

Notice that, contrary to what is stated in the original paper (at the end of Sec. IV), the PEF increases as the filter of the heralding photon becomes narrower and the filter of the HSP becomes broader. That is, according to Figs. 11 and 12 , if the idler is the HSP as in Figs. 11(a) and 12(a), large PEFs are achieved for small $\sigma_{s}$ and large $\sigma_{i}$. In the same way, if the signal is the HSP as in panels (b) of the figures, high PEF requires large $\sigma_{s}$ and small $\sigma_{i}$.

The authors thank E. Meyer-Scott for his crucial hint regarding the incorrect labels.
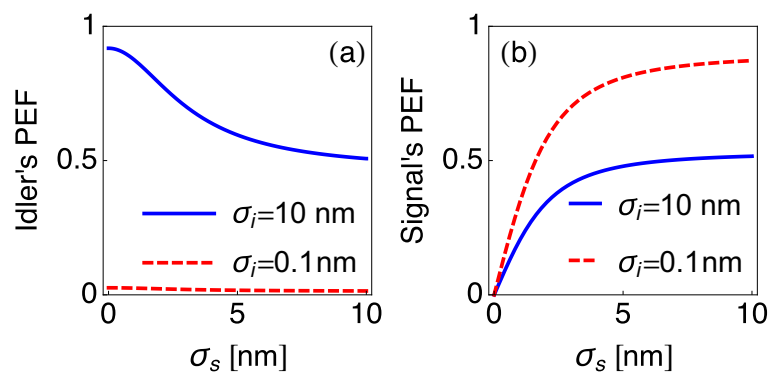

FIG. 11. Figure 11 in the original paper with the labels corrected. Spectral purity-efficiency factors (PEFs) for a specific value of the spectral correlation between signal and idler. The bandwidth and waist of the pump are $\sigma_{p}=1.0 \mathrm{~nm}$ and $w_{p}=10 \mu \mathrm{m}$, and the spatial-spectral correlation is present because the waist of the photons' spatial modes is $w_{s}=w_{i}=50 \mu \mathrm{m}$. We plot the idler's PEF when this photon is the heralded single photon (HSP) in (a) and when the signal is the heralded single photon in (b).
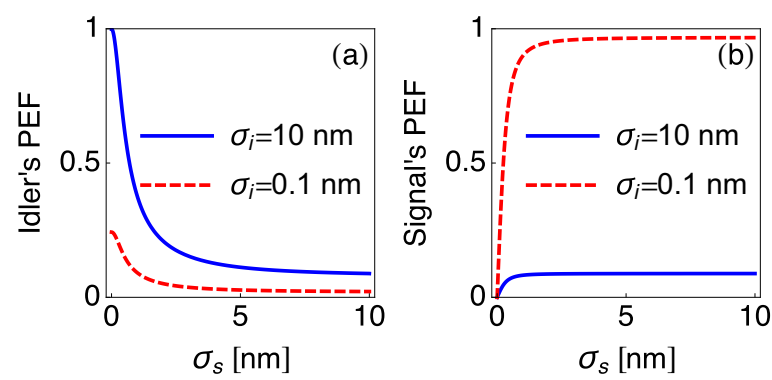

FIG. 12. Figure 12 in the original paper with the labels corrected. Spectral PEFs when the spectral signal-idler spectral correlation is stronger than in Fig. 11. The bandwidth and waist of the pump are $\sigma_{p}=0.1 \mathrm{~nm}$ and $w_{p}=10 \mu \mathrm{m}$, and the spatial-spectral correlation is present because the waist of the photons' spatial modes is $w_{s}=w_{i}=50 \mu \mathrm{m}$. We plot the idler's PEF when this photon is the HSP in (a) and when the signal is the heralded single photon in (b). 